\title{
AVALIAÇÃO DO TEOR DE ANTOCIANINAS EM POLPA DE ACEROLA CONGELADA PROVENIENTE DE FRUTOS DE 12 DIFERENTES ACEROLEIRAS (Malpighia emarginata D.C.) ${ }^{1}$
}

\author{
Vera Lúcia Arroxelas G. de LIMA,*, Enayde de A. MÉLO², \\ Maria Inês S. MACIEL ${ }^{2}$, Daisyvângela E. da Silva LIMA ${ }^{3}$
}

\section{RESUMO}

A ampla variabilidade genética nos pomares de aceroleiras brasileiros tem gerado frutos de coloração amarela a vermelha púrpura dificultando a obtenção de produtos de coloração avermelhada, cor esperada pelos consumidores. As antocianinas são pigmentos instáveis, responsáveis pela cor vermelha deste fruto maduro. Com o objetivo de determinar o teor destes pigmentos em polpa de acerola submetida ao congelamento foi instalado um experimento inteiramente casualisado. As polpas obtidas de frutos de 12 acessos (plantas) do Banco Ativo de Germoplasma da Universidade Federal Rural de Pernambuco (UFRPE) foram armazenadas a $-18^{\circ} \mathrm{C}$, por um periodo de 06 meses. Unidades amostrais de $30 \mathrm{~g}$ foram coletadas ao acaso, no periodo inicial e final do experimento, e submetidas a determinação quantitativa de antocianinas totais utilizando um método espectrofotométrico. Grande variação nos teores de antocianinas totais foi observada nos acessos estudados. No tempo zero de armazenamento o valor mínimo e máximo deste fitoquimico foi de $59,74 \mathrm{mg}$ e $3,79 \mathrm{mg} .100 \mathrm{~g}^{-1}$ de polpa, respectivamente. Após seis meses de congelamento foi constatada uma redução de 3,4\% a 23,6\% no teor desse pigmento nas amostras avaliadas. Evidenciou-se, portanto, que o armazenamento a $-18^{\circ} \mathrm{C}$ por seis meses reduziu o teor de antocianinas e que os pigmentos antociânicos dos acessos 08 e 13 apresentaram-se mais estáveis ao congelamento.

Palavras-chave: acerola; antocianinas; congelamento.

\section{SUMMARY}

EVALUATION OF TOTAL ANTHOCYANINS IN FROZEN ACEROLA PULP FROM FRUITS OF 12 DIFFERENT ACEROLA TREES (Malpighia emarginata D.C.). The great variability of the Brazilian acerola orchards results in fruits with yellow to red color, producing a light colored acerola pulp which is not as appealing as full red pulp. Anthocyanins are unstable pigments, responsible for red color in mature acerola. In order to examine some pigment degradation in frozen pulp acerola, it was installed a randomized experiment. Pulps obtained from 12 accesses (plants) of Germoplasm Active Bank from Federal Rural University of Pernambuco (UFRPE) were stored (-18 $\left.{ }^{\circ} \mathrm{C}\right)$ for 6 months. Total anthocyanins were determined in randomized samples $(30 \mathrm{~g})$ collected at the beginning and end of the experiment, using spectrophotometer method. Great variation on the total anthocyanin content was observed in the studied accesses. At time zero of

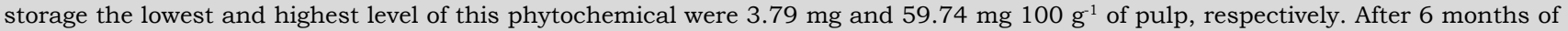
storage, a reduction of $3.4 \%$ to $23.6 \%$ on content of these pigments were shown. The storage at $-18^{\circ} \mathrm{C}$ for six months reduced the anthocyanin content. The anthocyanin pigments of accesses 08 and 13 were the most stable during frozen storage.

Keywords: acerola; anthocyanins; freezing.

\section{1 - INTRODUÇÃO}

A acerola, também conhecida como cereja-das-Antilhas (Malpighia emarginata D.C.), é um fruto avermelhado originário da região das Antilhas, norte da América do Sul e América Central [15]. Em função de seu alto teor de vitamina C (2.000 a $3.000 \mathrm{mg} / 100 \mathrm{~g}$ de suco), do elevado número de safras anuais ( 3 a 4 sob condições de sequeiros e 6 a 7 sob irrigação) e do potencial de exportação da polpa concentrada congelada, principalmente para o Japão, França, Inglaterra, Holanda e Estados Unidos, os plantios comerciais de acerola apresentaram em 1995 uma rápida expansão [20]. Na ocasião, estes autores vislumbraram um mercado francamente comprador pelos cinco anos seguintes, tornando potencialmente próspero este segmento da fruticultura brasileira.

A falta de padronização dos pomares em função da ampla variabilidade genética, decorrente da propagação da aceroleira por semente, tem gerado frutos de colora-

\footnotetext{
Recebido para publicação em 18/02/2000. Aceito para publicação em 23/03/2002.

${ }^{2}$ Universidade Federal Rural de Pernambuco/DCD, Av. Dom Manoel de Medeiros s/n, CEP: 52171-900,PE, e-mail:veraarroxelas@hotmail.com ${ }^{3}$ Bolsista PIBIC/CNPq-UFRPE.

* A quem a correspondência deve ser enviada.
}

ção amarela, amarela avermelhada, vermelha e vermelha púrpura [11]. SEMENSATO, PEREIRA [22] citam que a cor do suco de frutos de genótipos de aceroleira, cultivados sob elevada altitude (Município de Anápolis, GO), variou de amarela a vermelha. Há, portanto, o interesse em instalar pomares de aceroleira com germoplasma caracterizado e selecionado de forma a introduzir e difundir plantas que reúnam características de interesse agronômico, e dentre elas ressalta-se a produção de frutos de coloração vermelha [11]. Segundo ALVES et al. [2], embora os frutos de coloração amarela tenham características físico-químicas equivalentes aos vermelhos, estes são os preferidos pelos produtores e consumidores.

A cor vermelha da acerola, no estádio maduro, é devido à presença de antocianinas [6, 8]. Estes pigmentos são compostos fenólicos, solúveis em água, pertencentes ao grupo dos flavonóides [4], amplamente difundidos no reino vegetal, que conferem aos frutos, flores e raizes as nuanças de cores entre laranja e vermelha [9]. Em 05 seleções de acerola foi constatado uma variação no teor de antocianinas totais de 14,06 a $50,98 \mathrm{mg} 100 \mathrm{~g}^{-1}$ de polpa. A seleção de cor vermelha mais intensa apresentava o maior teor de antocianinas totais [14].

A Empresa Brasileira de Pesquisa Agropecuária (Embrapa), com base em seu Banco Ativo de 
Germoplasma de acerola, definiu a relação de descritores mínimos para este fruto. Nessa relação consta a cor vermelha como descritor da coloração da polpa do fruto maduro e, para a avaliação laboratorial, o teor total de antocianinas é considerado um dos dados químicos característicos da polpa. Dentre os 154 acessos deste banco, sete se destacam por apresentarem frutos de cor vermelha (película e polpa), além de outras características de interesse agronômico [19].

As antocianinas são pigmentos muito instáveis que podem ser degradadas, sob ação da vitamina $\mathrm{C}$, oxigênio, temperatura, $\mathrm{pH}$ do meio, entre outros, no próprio tecido ou destruídas durante o processamento e estocagem dos alimentos [5]. MESQUITA, VIGOA [18] ressaltaram que o alto teor de vitamina $\mathrm{C}$ da acerola e a presença de antocianinas, destacam este fruto no campo dos nutracêuticos, pela habilidade desses compostos em capturar radicais livres no organismo humano.

O congelamento, um dos principais métodos de conservação de frutos, tem sido bastante utilizado na conservação da acerola. Entretanto, ALVES et al. [1], evidenciaram que a cor vermelha destes frutos, ao serem congelados, foi modificada para amarela. A descoloração da polpa de acerola foi apontada por gerentes de produção e técnicos das empresas pernambucanas produtoras de polpas de frutas, como um problema que ocorre durante o seu processamento [3]. Assim, este trabalho teve como objetivo determinar o teor de antocianinas em polpas de acerolas congeladas, obtidas de frutos oriundos do Banco Ativo de Germoplasma da UFRPE (BAG - UFRPE).

\section{2 - MATERIAL E MÉTODOS}

O BAG-UFRPE, foi implantado seguindo um delineamento experimental constituído por blocos casualizados (5 blocos com 1 planta/bloco, totalizando 5 plantas do mesmo acesso), com 12 acessos (12 diferentes plantas), perfazendo um total de 60 plantas. Frutos $(500 \mathrm{~g})$ dos acessos, codificados pelos números $02,03,04,05,06$, $07,08,11,12,13,14$ e 15 , foram colhidos no estádio maduro, com coloração uniforme característica de cada acesso. O experimento foi instalado em delineamento inteiramente casualizado, com um tratamento e três repetições. Os frutos dos 05 blocos de cada acesso foram processados conjuntamente em centrífuga doméstica, as polpas obtidas distribuídas em potes plástico com capacidade de $30 \mathrm{~g}$ e armazenadas sob congelamento $\left(-18^{\circ} \mathrm{C}\right)$, em "freezer" doméstico, durante seis meses. Unidades amostrais de cada acesso foram retiradas ao acaso, no tempo zero e após seis meses de estocagem, para a determinação quantitativa de antocianinas totais, segundo método de LEES, FRANCIS [13]. Para esta quantificação, as polpas foram homogeneizadas com solução extratora (etanol 95\%: $\mathrm{HCl} 1,5 \mathrm{~N}-85: 15 \mathrm{v} / \mathrm{v}$ ) e estocadas por $12 \mathrm{~h} \mathrm{a} 4^{\circ} \mathrm{C}$. As amostras foram filtradas e os resíduos lavados exaustivamente com a solução extratora até a remoção completa dos pigmentos. Os filtrados foram coletados em balões volumétricos de $100 \mathrm{~mL}$, aferidos com a solução extratora, deixados em repouso no escuro por
$2 \mathrm{~h}$ a temperatura ambiente e a absorbância foi medida a $535 \mathrm{~nm}$. As determinações foram efetuadas em triplicata e os teores médios de antocianinas totais, referentes ao período inicial e final de armazenamento, foram comparados utilizando o Teste t de Student ao nível de 5\% de probabilidade.

\section{3 - RESULTADOS E DISCUSSÃO}

No tempo zero de armazenamento, as polpas provenientes dos frutos dos acessos estudados apresentaram teores de antocianinas totais que variaram de 3,79 a $59,74 \mathrm{mg} \cdot 100 \mathrm{~g}^{-1} \mathrm{e}$, conseqüentemente, diferentes tonalidade da cor vermelha. Os acessos 05 e 02 , destacaramse por terem apresentado, respectivamente, o mais alto e o mais baixo teor deste fitoquímico (Tabela 1). PAIVA et al. [21] também detectaram uma grande variação no teor de antocianinas totais em acerola $(1,97 \mathrm{a} 46,44 \mathrm{mg} / 100 \mathrm{~g})$. GONZAGA NETO, MATTUZ, SANTOS [10], relataram que polpas de acerola oriundas de 18 acessos apresentaram coloração que variou de amarela a vermelha-escura.

As antocianinas são glicosídios de antocianidinas, também chamadas de agliconas. Na natureza, 17 antocianidinas têm sido encontradas mas apenas 6 estão presentes em alimentos [9]. A diferença da cor dos vários frutos vermelhos depende da natureza e da concentração das antocianinas [12]. As várias tonalidades da cor vermelha das polpas produzidas, levam a crer que estão presentes diferentes concentrações de vários tipos de antocianinas. SILVA, GUEDES, MENEZES [24] verificaram que as antocianidinas presentes em dois cultivares de acerola eram malvidina e pelargonidina como frações principais e em outros dois cultivares predominavam malvidina, cianidina e pelargonidina. A cor das antocianinas é influenciada pelas condições do meio onde são encontradas. A presença de açúcares, de açúcares acilados, de grupos metoxil e hidroxil têm efeito marcante na cor e na reatividade das antocianinas [17].

TABELA 1. Antocianinas totais em polpa de acerola de 12 acessos armazenadas por 06 meses sob congelamento $\left(-18^{\circ} \mathrm{C}\right)$.

\begin{tabular}{cccc}
\hline Acessos & \multicolumn{2}{c}{ Antocianinas totais $\left(\mathrm{mg} \cdot 100 \mathrm{~g}^{-1}\right)^{*}$} & $\begin{array}{c}\text { Redução } \\
(\%)\end{array}$ \\
\cline { 2 - 3 } & \multicolumn{1}{c}{ Inicial } & final & 16,1 \\
02 & $3,79 \pm 0,10$ & $3,18 \pm 0,05$ & 14,8 \\
03 & $8,80 \pm 0,15$ & $7,50 \pm 0,11$ & 23,6 \\
04 & $23,22 \pm 0,40$ & $17,75 \pm 0,51$ & 14,9 \\
05 & $59,74 \pm 1,74$ & $50,81 \pm 1,04$ & 9,4 \\
06 & $18,88 \pm 0,24$ & $17,11 \pm 0,10$ & 16,2 \\
07 & $45,93 \pm 0,40$ & $38,49 \pm 0,30$ & 3,4 \\
08 & $48,54 \pm 0,33$ & $46,91 \pm 2,45^{* *}$ & 7,0 \\
11 & $36,66 \pm 1,01$ & $34,08 \pm 1,02$ & 9,6 \\
12 & $23,42 \pm 0,35$ & $21,18 \pm 0,20$ & 6,8 \\
13 & $37,07 \pm 1,37$ & $34,56 \pm 1,39^{* *}$ & 13,4 \\
14 & $28,00 \pm 0,36$ & $24,24 \pm 0,89$ & 17,6 \\
15 & $9,51 \pm 0,24$ & $7,84 \pm 0,13$ & \\
\hline
\end{tabular}

*os valores apresentados referem-se à média aritmética de três determinações **os valores não apresentam diferença significativa - Teste t Student ao nivel de $5 \%$ de probabilidade

No final do experimento, pode-se evidenciar uma redução de $3,4 \%$ a $23,6 \%$ nos teores de antocianinas totais (Tabela 1). As polpas dos frutos dos acessos 8 e 13 
foram as únicas cuja redução do teor desses pigmentos não foi estatisticamente significativa. Outros autores também detectaram redução de pigmentos antociânicos em acerola. SILVA, MENEZES, GUEDES [23], ao armazenarem polpa de acerola, sem tratamento térmico, sob congelamento por seis meses, evidenciaram um percentual de redução de 21,74\%. MATSUURA [16] detectou uma redução de 143,5 para $139,5 \mathrm{mg} / 1$ (expresso em malvidina 5-G) no teor de antocianinas em suco concentrado de acerola congelado $\left(-18^{\circ} \mathrm{C}\right)$ armazenado por 180 dias. Segundo CHEFTEL, CHEFTEL, BESANÇON [7], durante o congelamento as reações metabólicas são reduzidas, porém não totalmente inibidas, o que justifica a degradação dos pigmentos antociânicos nas polpas armazenadas a $-18^{\circ} \mathrm{C}$. A variação na redução do teor deste constituinte entre os diferentes acessos decorre das características intrínsecas dos frutos de cada planta.

\section{4 - CONCLUSÕES}

Os resultados permitem concluir que o armazenamento a $-18^{\circ} \mathrm{C}$ por seis meses reduziu o teor de antocianinas e que os pigmentos antociânicos dos acessos 08 e 13 apresentaram-se mais estáveis ao congelamento.

\section{5 - REFERÊNCIAS}

[1] ALVES, R.E.; CHITARRA, A.B.; FREIRE, D.C.; SOUZA, K.R.; SIQUEIRA, S.M.P. Yellowing of frozen acerola (Malpighia emarginata) fruit. Proceedings of Interamerican Society for Tropical Horticulture, Guatemala, v. 41, p. 199204, 1997.

[2] ALVES, R.E; FILGUEIRAS, H.A.C.; MOSCA, J.L.; MENEZES, J.B. Brazilian experience on the handling of acerola fruits for international trade: harvest and postharvest recommendations. Acta Horticulturae, v.485, p.31-36, 1999.

[3] BASTOS, M.S.R.; OLIVEIRA, M.E.B.; FEITOSA, T. Perfil tecnológico da produção da polpa da fruta congelada no Estado de Pernambuco. XVI Congresso Brasileiro de Ciência e Tecnologia de Alimentos, Rio de Janeiro, RJ, 15-17 de julho de 1998.

[4] BOBBIO, P.A.; BOBBIO, F.O. Pigmentos naturais. In: BOBBIO, P.A.; BOBBIO, F.O.(Ed.) Introdução à Quimica de Alimentos. São Paulo: Varela, 1995. Cap. 6, p. 191-232

[5] BROUILLARD, R. Chemical structure of anthocyanins. In: MARKAKIS, P.(Ed) Anthocyanins as food colors. London: Academic Press, 1982. Cap. 1, p. 1-40

[6] CHAN JR., H. T.; YAMAMOTO, H. Y. Kinetics of anthocyanin decomposition in acerola juice. ASEAN Food Journal, v. 9, n. 4, p. 132-135, 1994.

[7] CHEFTEL, J.C.; CHEFTEL, H.; BESANÇON, P. Métodos de conservacion. In: CHEFTEL, J.C.; CHEFTEL, H.; BESANÇON, P.(Ed.) Introduccion a la bioquímica y tecnologia de los alimentos. Zaragoza: Acribia, 1983. v. 2, Cap.7, p.173-202

[8] CONCEIÇÃO, M. P. J. Cinética de degradação térmica de antocianinas em suco de acerola (Malpighia glabra L.). Viçosa, 1997. 59p. Dissertação de Mestrado- Universidade Federal de Viçosa (UFV).
[9] FRANCIS, F.J. Food colorants: anthocyanins. Critical Reviews in Food Science and Nutrition, v. 28, n. 4, p. 273-314, 1989.

[10] GONZAGA NETO, L.; MATTUZ, B-H; SANTOS, C.A.F. Caracterização agronômica de clones de aceroleira (Malpighia spp) na região do submédio São Francisco. Revista Brasileira de Fruticultura, v. 21,n. 2, p. 110115, 1999.

[11] GONZAGA NETO, L. Melhoramento genético da aceroleira. In: SÃO JOSÉ, A.R., ALVES, R.E. (Ed.) Acerola no Brasil- produção e mercado. Vitória da Conquista: DFZ/ UESB, 1995. p. 15-27

[12] GROSS, J. Anthocyanins. In: GROSS, J. (Ed.) Pigments in fruits. London: Academic Press, p.59-85, 1987.

[13] LEES, D.H.; FRANCIS, F.J. Standardization of pigment analyses in cranberries. HortScience, v. 7, n. 1, p.8384, 1972.

[14] LIMA, V.L.A.G.; MÉLO, E.A.; LIMA, L.S.; NASCIMENTO, P.P. Flavonóides em seleções de acerola (Malpighia sp. L.). 1- Teor de antocianinas e flavonóis totais. Ciência Rural, v. 30, n. 6, p. 1063-1064, 2000.

[15] MARINO NETTO, L. 1986. Acerola, a cereja tropical. Nobel, São Paulo.

[16] MATSUURA, F.C.A.U. Processamento e caracterização de suco integral e concentrado congelado de acerola. Campinas, 1994. 142p. Dissertação de Mestrado - Faculdade de Engenharia de Alimentos, .Universidade Estadual de Campinas (UNICAMP).

[17] MAZZA, G.; BROUILLARD, R. Recent developments in the stabilization of anthocyanins in food products. Food Chemistry, v. 25, p. 207-225, 1987.

[18] MESQUITA, P.C.; VIGOA, Y.G. La acerola. Fruta marginada de America con alto contenido de acido ascorbico. Alimentaria, v. 37, n. 309, p.113-125, 2000.

[19] OLIVEIRA, J.R.P.; SOARES FILHO, W.S.; NASCIMENTO, A.S.; COSTA, D.C.; MATSUURA, F.C.A.U.; GOMES, J.C.; CARVALHO, J.E.B.; REINAHRDT, D.H.; OLIVEIRA, R.P. Programa de pesquisa de acerola: Embrapa mandioca e fruticultura. EMBRAPA-CNPMF. Documentos 75, 1998.

[20] OLIVEIRA, J.R.P.; SOARES FILHO, W.S. Acerola: conservação, caracterização e seleção de germoplasma pelo CNPMF - EMPRAPA. In: Acerola no Brasil - produção e Mercado. SÃO JOSÉ, A. R.; ALVES, R. E. Ed. Vitória da Conquista - BA, DFZ/UESB, p. 22-27, 1995.

[21] PAIVA, J.R.; CORDEIRO, E.R.; ALVES, R.E.; ALMEIDA, A.S.; PINTO, S.A.A. Seleção e clonagem de plantas de acerola. XIV Encontro de Genética do Nordeste, Recife, PE, 02-05 de junho de 1999.

[22] SEMENSATO, L.R.; PEREIRA, A.S. Características de frutos de genótipos de aceroleira cultivados sob elevada altitude. Pesquisa Agropecuária Brasileira, v. 35, n. 12, p. 2529-2536, 2000.

[23] SILVA, M.F.V.; MENEZES, H.C.; GUEDES, M. C. Efeito de diferentes tratamentos térmicos sobre as antocianinas na polpa de acerola. III Simpósio Latino Americano de Ciência de Alimentos, Campinas, SP, 16-19 de novembro de 1999.

[24] SILVA, M.F.V.; GUEDES, M.C.; MENEZES, H.C. Caracterização dos pigmentos antociânicos de diferentes cultivares de acerola (Malpighia glabra) por CLAE. VII Congresso Latino-Americano de Cromatografia, Águas de São Pedro, SP, 25-27 de março de 1998. 